\title{
COORDINATIZATION OF ORTHOCOMPLEMENTED AND ORTHOMODULAR POSETS
}

\author{
S. P. GUDDER AND R. H. SCHELP
}

\begin{abstract}
Generalizations of Baer *-semigroups called partial Baer *-semigroups and OM-partial Baer *-semigroups are introduced. It is shown that the set of closed projections of a (OM) partial Baer *-semigroup form an (orthomodular) orthocomplemented poset. Conversely (orthomodular) orthocomplemented posets are coordinatized by $(\mathrm{OM})$ partial Baer *-semigroups. It is shown that these coordinatizing semigroups are minimal.
\end{abstract}

1. Introduction. David Foulis [1] has shown that an orthomodular lattice $L$ can be coordinatized by a Baer *-semigroup $S$. By this it is meant that $L$ is isomorphic to the set of closed projections in $S$. Conversely, it is shown in [1] that the set of closed projections in a Baer *-semigroup forms an orthomodular lattice. Since the time of this result it has been an open question whether a similar coordinization can be given for orthomodular, or still more generally, orthocomplemented posets. This question not only has mathematical interest, but it is important in the axiomatic foundations of quantum mechanics where it is generally agreed that quantum proposition systems form an orthomodular poset. In this paper we prove coordinatization theorems for orthocomplemented and orthomodular posets in terms of a generalization of Baer *-semigroups which we call partial Baer *-semigroups. We also show that in the present situation there are uniqueness conditions holding. The method of coordinatization is quite different from Foulis' work and is based on a rather simple construction.

2. Partial Baer *-semigroups. Let $S$ be a set and $R \subseteq S \times S$ a relation on $S .(S, R)$ is called a partial semigroup if there is a map from $R$ into $S$ denoted by $(x, y) \rightarrow x y$ with the following property: for any $x, y, z \in S$ such that $(x, y),(y, z) \in R$ then $(x, y z) \in R$ if and only if $(x y, z) \in R$ and in that case $(x y) z=x(y z)$. For conciseness we usually omit the $R$ and call $S$ a partial semigroup. Also for brevity in presentation, products $x y$ may appear in expressions without specific mention that $(x, y) \in R$. A partial semigroup $S$ is a partial involution semigroup if there is a map ${ }^{*}$ from $S$ into $S$ (called an involution)

Received by the editors July 30, 1969.

AMS Subject Classifications. Primary 0620, 0630, 0635, 0670; Secondary 8106.

Key Words and Phrases. Partially ordered sets, orthocomplemented posets, orthomodular posets, Baer *-semigroups, coordinatization theorems. 
such that $x=x^{* *}$ for all $x \in S$ and if $(x, y) \in R$ then $\left(y^{*}, x^{*}\right) \in R$ with $(x y)^{*}=y^{*} x^{*}$. A zero is an element $o$ in $S$ which satisfies $(x, o) \in R$ for all $x \in S$ and $x o=o$. Notice that $o^{*} o=o$ so $o^{*}=\left(o^{*} o\right)^{*}=o^{*} o^{* *}$ $=o^{*} o=o$ from which $(o, x) \in R$ for every $x \in S$ and $o x=o$. Also if a zero exists, it is unique. An element $e \in S$ is a projection if $(e, e) \in R$ and $e=e e=e^{*}$. The projections of $S$ will be denoted by $P(S)$. A partial involution semigroup $S$ with zero is a partial Baer *-semigroup if for every $x \in S$ there is a projection $x^{\prime} \in P(S)$ satisfying property (B):

$$
\left(x^{\prime}, y\right) \in R \text { and } x^{\prime} y=y \text { if and only if }(x, y) \in R \text { and } x y=0 .
$$

It is straightforward to show that the projection $x^{\prime}$ is unique.

Notice that a partial Baer *-semigroup is a generalization of a Baer *-semigroup. In fact, $S$ is a Baer *-semigroup if and only if $R=S \times S$. For an example of a partial Baer *-semigroup that is not a Baer *-semigroup, let $S$ be the set of all rectangular matrices. Identify all the rectangular matrices that have only zero entries and let this class be the zero element. Define multiplication by zero in the natural way and $R$ becomes the set of pairs of matrices that can be multiplied, ${ }^{*}$ the transpose operation, and $x^{\prime}$ the projection into the null space of the matrix $x^{*}$.

In the sequel $S=\left(S, R,{ }^{*},{ }^{\prime}\right)$ will denote a partial Baer *-semigroup. We say that $e \in P(S)$ is a closed projection if $e=e^{\prime \prime}$ and denote this set by $P^{\prime}(S)$. If $e, f \in P(S)$ define $e \leqq f$ if $(e, f) \in R$ and $e f=e$. By taking involutions it follows that $e \leqq f$ if and only if $(f, e) \in R$ and $f e=e$.

Lemma 2.1. Let $e, f \in P(S)$. (i) $e \leqq e^{\prime \prime}$; (ii) $e \leqq f$ implies $f^{\prime} \leqq e^{\prime}$; (iii) $e^{\prime} \in P^{\prime}(S)$; (iv) $(P(S))^{\prime}=P^{\prime}(S)$.

Proof. (i) Since $x^{\prime} x^{\prime}=x^{\prime}$ we have by property (B) $\left(x, x^{\prime}\right) \in R$ with $x x^{\prime}=o$ for every $x \in S$. Therefore $o=o^{*}=x^{\prime} x^{*}$ which implies $x^{\prime \prime} x^{*}=x^{*}$ so $x=x x^{\prime \prime}$. In particular $e=e e^{\prime \prime}$ for every $e \in P(S)$ or $e \leqq e^{\prime \prime}$. (ii). It follows from $e \leqq f$ and $f f^{\prime}=o$ that $(e, f),\left(f, f^{\prime}\right)$ and $(e, o)=\left(e, f f^{\prime}\right) \in R$. Therefore $\left(e f, f^{\prime}\right) \in R$ and $o=e o=e\left(f f^{\prime}\right)=(e f) f^{\prime}=e f^{\prime}$ and hence $\left(e^{\prime}, f^{\prime}\right) \in R$ with $f^{\prime}=e^{\prime} f^{\prime}$. Thus $f^{\prime} \leqq e^{\prime}$. The remaining parts of the lemma are easily established.

Lemma 2.2. Let $e \in P^{\prime}(S), x \in S$; then $(x, e) \in R$ and $x=x e$ if and only if $x^{\prime \prime} \leqq e$; that is, $x^{\prime \prime}$ is the smallest closed projection that acts as a right unit for $x$. In particular $x=x x^{\prime \prime}$.

Proof. First notice that $x^{\prime \prime} \in P^{\prime}(S)$ by (iv) of Lemma 2.1. Let $(x, e) \in R$ with $x=x e$ so that we obtain $o=x o=x\left(e e^{\prime}\right)=(x e) e^{\prime}=x e^{\prime}$. Hence $e^{\prime}=x^{\prime} e^{\prime}$ from which $e^{\prime} \leqq x^{\prime}$ and $x^{\prime \prime} \leqq e^{\prime \prime}=e$. Conversely since 
we established $x=x x^{\prime \prime}$ in the proof of Lemma 2.1 (i), whenever $x^{\prime \prime} \leqq e$ we obtain $x=x x^{\prime \prime}=x\left(x^{\prime \prime} e\right)=\left(x x^{\prime \prime}\right) e=x e$.

CoRollary 2.3. If $x, y \in S$ with $(x, y) \in R$ then $(x y)^{\prime \prime} \leqq y^{\prime \prime}$.

Proof. $x y=x\left(y y^{\prime \prime}\right)=(x y) y^{\prime \prime}$ which implies $(x y)^{\prime \prime} \leqq y^{\prime \prime}$.

Corollary 2.4. If $x \in S$ then $x^{\prime}=x^{\prime \prime \prime}$.

Proof. Since $x^{\prime \prime} x^{\prime \prime \prime}=o$ we have $o=x\left(x^{\prime \prime} x^{\prime \prime \prime}\right)=\left(x x^{\prime \prime}\right) x^{\prime \prime \prime}=x x^{\prime \prime \prime}$. Hence, $x^{\prime} x^{\prime \prime \prime}=x^{\prime \prime \prime}$ and $x^{\prime \prime \prime} \leqq x^{\prime}$. The reverse inequality follows from Lemma 2.1 (i).

Let $P=\{a, b, c, \cdots\}$ be a partially ordered set with first and last elements $o$ and 1 respectively. Recall that $P$ is an orthocomplemented poset if for every $a \in P$ there is an $a^{\prime} \in P$ such that (i) $a^{\prime \prime}=a$, (ii) $a \leqq b$ implies $b^{\prime} \leqq a^{\prime}$, (iii) inf $\left\{a, a^{\prime}\right\}=o$.

TheOREM 2.5. $\left(P^{\prime}(S), \leqq,^{\prime}\right)$ is an orthocomplemented poset.

Proof. We first show that $\leqq$ is a partial order on $P(S)$, hence also on $P^{\prime}(S)$. Clearly $e \leqq e$ for all $e \in P(S)$. Also if $e \leqq f$ and $f \leqq e$ then $e=e f=f$. Finally if $e, f, g \in P(S)$ with $e \leqq f, f \leqq g$ then $(e, f),(f, g)$, $(e, f g) \in R$ which imply $(e f, g) \in R$ and $e=e f=e(f g)=(e f) g=e g$ or that $e \leqq g$. By Lemma $2.1^{\prime}$ is a map from $P^{\prime}(S)$ into $P^{\prime}(S)$ with $e=e^{\prime \prime}$ for all $e \in P^{\prime}(S)$ and $e \leqq f$ in $P^{\prime}(S)$ implies $f^{\prime} \leqq e^{\prime}$. Next notice that if $f, e \in P(S)$ with $f \leqq e, e^{\prime}$ then $(f, e),\left(f, e^{\prime}\right) \in R$ and $f=f e^{\prime}=(f e) e^{\prime}$ $=f\left(e e^{\prime}\right)=f o=o$. Hence, since $o o=o^{*}=o$, we have $o \in P(S)$ and $\inf _{P(S)}\left\{e, e^{\prime}\right\}=o$. Furthermore, since $o o^{\prime}=o$ we have $o \leqq o^{\prime}$ which implies $o^{\prime \prime} \leqq o^{\prime}=o^{\prime \prime \prime}$. Thus $o^{\prime \prime} \leqq o^{\prime \prime}, o^{\prime \prime \prime}$ which implies that $o^{\prime \prime}=o$ or that $o \in P^{\prime}(S)$. It follows that if $e \in P^{\prime}(S)$ then $\inf _{P^{\prime}(S)}\left\{e, e^{\prime}\right\}=o$. Therefore $\left(P^{\prime}(S), \leqq,^{\prime}\right)$ is an orthocomplemented poset with first and last elements $o$ and $o^{\prime}$ respectively.

Henceforth we shall let $\wedge$ and $\vee$ denote the infimum and supremum respectively (when they exist) of pairs of elements of a poset $P$. If the poset $P$ under discussion is not clear, we shall write $\wedge_{P}$ or $\bigvee_{P}$. We next establish some important sufficient conditions for the existence of the infimum of two projections.

Lemma 2.6. (i) If $e, f \in P(S)$ satisfy $(e, f) \in R$ and $e f=f e$, then $e \wedge P(S) f=e f$. (ii) If $e, f \in P^{\prime}(S)$ satisfy $(e, f) \in R$ and $e f=f e$, then $e f \in P^{\prime}(S)$ and $e f=e \wedge_{P(S)} f=e \wedge_{P^{\prime}(S)} f$.

Proof. (i) First notice that $e f=(e e)(f f)=[(e e) f] f=[e(e f)] f=[e(f e)] f$ $=(e f)(e f)$ and $(e f)^{*}=f e=e f$ which imply $e f \in P(S)$. In addition $e f=e(f f)=(e f) f$ and $e f=(e e) f=e(e f)=(e f) e$ so $e f \leqq f$, $e$. Finally if $g \in P(S)$ and $g \leqq e, f$ then $g=g f=(g e) f=g(e f)$ or $g \leqq e f$. (ii) Since 
$e f \leqq e, f$, by Lemma 2.1 (ii) $(e f)^{\prime \prime} \leqq e, f$ and hence by (i) $(e f)^{\prime \prime} \leqq e f$. Applying Lemma 2.1 (i) $(e f) \leqq(e f)^{\prime \prime}$ and hence $e f \in P^{\prime}(S)$. It follows from (i) that $e f=e \wedge_{P^{\prime}(S)} f$.

Lemma 2.7. If $e, f \in P(S)$ and $(e, f),\left(e, f^{\prime}\right) \in R$ with $e f=f e$, then $e f^{\prime}=f^{\prime} e$.

Proof. $o=e o=e\left(f f^{\prime}\right)=(e f) f^{\prime}=(f e) f^{\prime}=f\left(e f^{\prime}\right)$ which implies $\left(e f^{\prime}\right)$ $=f^{\prime}\left(e f^{\prime}\right)$. Hence $\left(e f^{\prime}\right)^{*}=f^{\prime} e=\left[f^{\prime}\left(e f^{\prime}\right)\right]^{*}=f^{\prime}\left(e f^{\prime}\right)=e f^{\prime}$.

Recall that an orthocomplemented poset $P=\{a, b, c, \cdots\}$ is orthomodular if the orthomodular law, $a \leqq b$ implies $b=a \vee\left(b \wedge a^{\prime}\right)$, holds. We will show in the next section that $P^{\prime}(S)$ need not be orthomodular so it is of interest to find a condition on $S$ which is sufficient for $P^{\prime}(S)$ to be orthomodular. We thus give such a condition. $S$ is said to be an OM-partial Baer *-semigroup if it satisfies the following:

$(\mathrm{OM}) \quad$ if $e, f \in P^{\prime}(S)$ and $e \leqq f$ then $\left(e^{\prime}, f\right) \in R$.

In the next section we will show that every orthomodular poset can be coordinatized by an $S$ satisfying this condition.

Lemma 2.8. Let $S$ be an OM-partial Baer *-semigroup and $e$, $f \in P^{\prime}(S)$. (i) If $\left(e^{\prime}, f\right) \in R$, then $\left(\left(e^{\prime} f\right)^{\prime}, f\right) \in R$ and $e \wedge f=\left(e^{\prime} f\right)^{\prime} f$. (ii) If $(e, f) \in R$, then $\left(e \bigvee f^{\prime}\right) \wedge f=(e f)^{\prime \prime}$.

Proof. (i) $o=e^{\prime} o=e^{\prime}\left(f f^{\prime}\right)=\left(e^{\prime} f\right) f^{\prime}$ so that $f^{\prime}=\left(e^{\prime} f\right)^{\prime} f^{\prime}$ which implies $f^{\prime} \leqq\left(e^{\prime} f\right)^{\prime}$. By $(\mathrm{OM})\left(\left(e^{\prime} f\right)^{\prime}, f\right) \in R$ and hence by Lemmas 2.6 and 2.7 $f\left(e^{\prime} f\right)^{\prime}=\left(e^{\prime} f\right)^{\prime} f=\left(e^{\prime} f\right)^{\prime} \wedge f$. Now suppose $q \in P^{\prime}(S)$ and $q \leqq\left(e^{\prime} f\right)^{\prime}, f$. Then $q=q\left(e^{\prime} f\right)^{\prime}=\left(e^{\prime} f\right)^{\prime} q$ which implies $o=\left(e^{\prime} f\right) q=e^{\prime}(f q)=e^{\prime} q$ and $q=e^{\prime \prime} q=e q$ so $q \leqq e$. In particular $\left(e^{\prime} f\right)^{\prime} f \leqq e, f$. Next suppose $r \in P^{\prime}(S)$ and $r \leqq e, f$. Then $r=e^{\prime \prime} r$ and hence $o=e^{\prime} r=e^{\prime}(f r)=\left(e^{\prime} f\right) r$. It follows that $\left(e^{\prime} f\right)^{\prime} r=r$ so $r \leqq\left(e^{\prime} f\right)^{\prime}$. Thus $e \wedge f=\left(e^{\prime} f\right)^{\prime} f$. (ii) $(e, f) \in R$ implies by (i) that $e^{\prime} \wedge f=(e f)^{\prime} f$ from which $e \bigvee f^{\prime}=\left[(e f)^{\prime} f\right]^{\prime}$. Also $\left(e \bigvee f^{\prime}\right)^{\prime} \leqq f$ so that $\left(\left(e \vee f^{\prime}\right)^{\prime}, f\right) \in R$ and by (i) $\left(e \vee f^{\prime}\right) \wedge f=\left\{\left[(e f)^{\prime} f\right] f\right\}^{\prime} f=\left[(e f)^{\prime} f\right]^{\prime} f$. By Corollary $2.3(e f)^{\prime \prime} \leqq f$ so by $(\mathrm{OM})\left((e f)^{\prime \prime \prime}, f\right) \in R$. Hence by (i) $(e f)^{\prime \prime}=(e f)^{\prime \prime} \wedge f=\left[(e f)^{\prime \prime \prime} f\right]^{\prime} f=\left[(e f)^{\prime} f\right]^{\prime} f$ where the last equality follows from Corollary 2.4. Hence $(e f)^{\prime \prime}=\left(e \bigvee f^{\prime}\right) \wedge f$.

Theorem 2.9. If $S$ is an OM-partial Baer *-semigroup, then $\left(P^{\prime}(S), \leqq,^{\prime}\right)$ is an orthomodular poset.

Proof. We need only show that whenever $e \leqq f$ then $e \bigvee\left(f \wedge e^{\prime}\right)=f$. When $e \leqq f$ we have $f^{\prime} \leqq e^{\prime}$ so that $\left(f^{\prime}, e^{\prime}\right) \in R$. Hence by Lemma 2.8 (ii) $\left(f^{\prime} \bigvee e\right) \wedge e^{\prime}=\left(f^{\prime} e^{\prime}\right)^{\prime \prime}=f^{\prime}$ and $e \bigvee\left(f \wedge e^{\prime}\right)=f$. 
We will show in the next section that $(\mathrm{OM})$ is not a necessary condition for $P^{\prime}(S)$ to be orthomodular.

3. Coordinatization theorems. In the previous section we established only the first part of our primary result, namely that the closed projections of any partial Baer *-semigroup (OM-partial Baer *-semigroup) forms an orthocomplemented (orthomodular) poset. We also want the converse, that given any orthocomplemented (orthomodular) poset $P$ one can find a partial Baer *-semigroup (OM-partial Baer *-semigroup) which coordinatizes $P$, i.e., one whose closed projections are order isomorphic in both directions with $P$ with the isomorphism preserving orthocomplementation.

We first coordinatize an orthocomplemented poset $P$. If $a, b \in P$ are related (i.e., $a \leqq b$ or $b \leqq a$ ) we write $a \mathrm{r} b$ and if $a$ and $b$ are orthogonal (i.e., $a \leqq b^{\prime}$ ) we write $a \perp b$.

TheOREM 3.1. If $P$ is an orthocomplemented poset then there is a partial Baer *-semigroup $S_{0}(P)$ such that $P$ is isomorphic to $P^{\prime}\left(S_{0}(P)\right)$.

Proof. Let $\delta_{0}(P)=P$ and let $R=\{(a, b): a \mathrm{r} b$ or $a \perp b\}$. If $(a, b) \in R$ define $a b=a \wedge b$. We first show that $\left(\mathcal{S}_{0}(P), R\right)$ is a partial semigroup. To do this it will suffice to show $(a, b),(b, c)$ and $(a b, c) \in R$ implies $(a, b c) \in R$ since then $a(b c)=a \wedge b \wedge c=(a b) c$. We consider the following cases:

(1) $a \mathrm{r} b, b \mathrm{r} c,(a \wedge b) \mathrm{r} c$. If $a \leqq b$ and $a \leqq c$ then $a \leqq b \wedge c$ and $a \mathrm{r}(b \wedge c)$. If $a \leqq b$ and $c \leqq a$ then $c \leqq a \leqq b$ giving $b \wedge c \leqq a$ and $a \mathrm{r}(b \wedge c)$. If $b \leqq a$ and $b \leqq c$ then $b \wedge c \leqq a$ and $a \mathrm{r}(b \wedge c)$. Finally, if $b \leqq a$ and $b \geqq c$, then $c \leqq b \leqq a$ giving $b \wedge c \leqq a$ and $a \mathrm{r}(b \wedge c)$.

(2) $a \mathrm{r} b, b \mathrm{r} c,(a \wedge b) \perp c$. If $a \leqq b$ then $a \perp c$ and hence $a \perp(b \wedge c)$. If $b \leqq a$ then $b \perp c$ giving $b \wedge c=0$ and $a \perp(b \wedge c)$.

(3) $b \perp c$. In this case $b \wedge c=0$ and $a \mathrm{r}(b \wedge c)$.

(4) $a \perp b$. In this case $a \perp(b \wedge c)$.

Define $a^{*}=a$ for all $a \in P$. Certainly $a^{* *}=a$ and if $(a, b) \in R$ then $\left(b^{*}, a^{*}\right) \in R$ and $(a b)^{*}=b^{*} a^{*}$. It is clear that 0 is a zero for $\delta_{0}(P)$. For $a \in S_{0}(P)$ define the projection corresponding to $a$ in condition (B) by $a^{\prime}$. We now show that $\S_{0}(P)=\left(S_{0}(P), R,{ }^{*},{ }^{\prime}\right)$ is a partial Baer *-semigroup. If $\left(b^{\prime}, a\right) \in R$ and $b^{\prime} a=a$ we have $b^{\prime} \wedge a=a$ and so $a \perp b$. Hence $(b, a) \in R$ and $b a=0$. Conversely, if $(b, a) \in R$ and $b a=0$, then $b \wedge a=0$. If $a \mathrm{r} b$ and $a=0$ then $\left(b^{\prime}, a\right) \in R$ and $b^{\prime} a=a$. If $a \mathrm{r} b$ and $b=0$ then again $\left(b^{\prime}, a\right) \in R$ and $b^{\prime} a=a$. Finally if $a \perp b$ then $\left(b^{\prime}, a\right) \in R$ and $b^{\prime} a=b^{\prime} \wedge a=a$. Notice that every element of $\varsigma_{0}(P)$ is a closed projection so that $P^{\prime}\left(S_{0}(P)\right)=S_{0}(P)$. It is clear that $P$ is isomorphic to $S_{0}(P)$ under the identity map $a \rightarrow a$. 
We call a partial Baer *-semigroup $S$ for which $P^{\prime}(S)$ is isomorphic to $P$ a coordinatizing partial Baer ${ }^{*}$-semigroup for $P$. Notice that we have actually proved the stronger result that an orthocomplemented poset can be coordinatized by a partial Baer *-semigroup of projections.

At this point it might be well to note that Theorem 3.1 clarifies two comments made in the preceding section. First if one considers a nonorthomodular, orthocomplemented poset $P$ and any coordinatizing partial Baer *-semigroup $S$ for $P$ then $P^{\prime}(S)$ cannot be orthomodular. Hence the closed projections of an arbitrary partial Baer *-semigroup need not form an orthomodular poset. Secondly, it is easy to see that a necessary and sufficient condition for $\delta_{0}(P)$ in Theorem 3.1 to satisfy (OM) is that $a, b \in P$ with $a \leqq b$ implies $a=0$ or $b=1$ or $a=b$. Thus condition (OM) is not a necessary condition for the closed projections of a partial Baer *-semigroup to satisfy the orthomodular identity. In fact, it appears quite difficult to obtain an algebraic condition on an arbitrary Baer *-semigroup $S$ which would be both necessary and sufficient for $P^{\prime}(S)$ to be orthomodular.

We shall next coordinatize orthomodular posets with OM-partial Baer *-semigroups. It is clear from the second comment in the previous paragraph that the construction in Theorem 3.1 will not do. We accomplish this by using the same construction with a stronger relation than $R$ of Theorem 3.1.

For the remainder of this section, $P$ will be an orthomodular poset. We say that $a, b \in P$ are compatible, written $a \mathfrak{e} b$, if there exist elements $a_{1}, b_{1}, c \in P$ which are mutually orthogonal with $a=a_{1} \bigvee c$ and $b=b_{1} \bigvee c$. Clearly if $a$ and $b$ are related or orthogonal then $a \mathfrak{e} b$. It can be shown [3], [4] that $a \mathfrak{C} b$ if and only if $a$ and $b$ are contained in a common Boolean subalgebra of $P$. Hence if $a \mathrm{e} b$ then $a \mathfrak{e} b^{\prime}$, all inf's and sup's exist among $a, b, a^{\prime}$ and $b^{\prime}$, and the distributive laws hold for these elements. We will use these facts without further comment.

Theorem 3.2 (Cf. [2]). (1) Let $a, b \in P$ with $a \wedge b, a^{\prime} \wedge b$ and $a \wedge b^{\prime}$ all existing. Then the following are equivalent: (i) $a \mathfrak{e} b$, (ii) $a=(a \wedge b)$ $\vee\left(a \wedge b^{\prime}\right)$, (iii) $a \wedge b=a \wedge\left(b \bigvee a^{\prime}\right)$. (2) Let $a, b, c \in P$ with $a \bigvee b, a \wedge c$, $b \wedge c,(a \bigvee b) \wedge c$, and $(a \wedge c) \vee(b \wedge c)$ all existing in $P$. If two of the relations $a \mathfrak{e} b, b$ e $c, a$ e $c$ hold then $(a \bigvee b) \wedge c=(a \wedge c) \bigvee(b \wedge c)$

The next lemma has been proved independently by several mathematicians; however, as far as we know, this is the first time it has been published. 
Lemma 3.3. If $a, b, c \in P, a \mathfrak{e} b, b \mathfrak{e} c$ and $a \mathfrak{e}(b \wedge c)$ then $(a \wedge b) \mathfrak{e} c$.

Proof. Applying Lemma $3.2(1)$ we need only show that $(a \wedge b) \wedge c$, $(a \wedge b)^{\prime} \wedge c,(a \wedge b) \wedge c^{\prime}$ exist and that $a \wedge b=\left[(a \wedge b) \wedge c^{\prime}\right] \vee[(a \wedge b) \wedge c]$. Since $a \mathfrak{C}(b \wedge c)$ we have $a \wedge(b \wedge c)=(a \wedge b) \wedge c$ existing. Since $a \wedge(b \wedge c) \leqq a \wedge b$ we have $(a \wedge b) \wedge(a \wedge b \wedge c)^{\prime}$ exists. Hence $(a \wedge b)$ $\wedge(a \wedge b \wedge c)^{\prime}=b \wedge\left[a \wedge\left(a^{\prime} \backslash[b \wedge c]^{\prime}\right)\right]=b \wedge\left[a \wedge(b \wedge c)^{\prime}\right]=a \wedge\left[b \wedge\left(b^{\prime} \vee c^{\prime}\right)\right]$ $=a \wedge\left(b \wedge c^{\prime}\right)=(a \wedge b) \wedge c^{\prime}$. By Lemma 3.2 (1) $b$ e $c$ implies $b=\left(b \wedge c^{\prime}\right)$ $\vee(b \wedge c)$. Therefore by Lemma 3.2 (2) $a \wedge b=a \wedge\left[\left(b \wedge c^{\prime}\right) \bigvee(b \wedge c)\right]$ $=\left[a \wedge\left(b \wedge c^{\prime}\right)\right] \vee[a \wedge(b \wedge c)]=\left[(a \wedge b) \wedge c^{\prime}\right] \vee[(a \wedge b) \wedge c]$. Finally, we have that $\left[c^{\prime} \bigvee(a \wedge b \wedge c] \bigvee\left(a \wedge b \wedge c^{\prime}\right)=c^{\prime} \vee\left[(a \wedge b \wedge c) \bigvee\left(a \wedge b \wedge c^{\prime}\right)\right]\right.$ exists and using the previous sentence this last expression is $c^{\prime} \bigvee(a \wedge b)$. This implies $(a \wedge b)^{\prime} \wedge c=\left[c^{\prime} \vee(a \wedge b)\right]^{\prime}$ exists.

We are now ready to obtain the coordinatization of an orthomodular poset.

THEOREM 3.4. If $P$ is an orthomodular poset then there is an OMpartial Baer *-semigroup $S_{1}(P)$ such that $P^{\prime}\left(S_{1}(P)\right)$ is isomorphic to $P$.

Proof. Let $S_{1}(P)=P$ and let $R=\{(a, b): a$ e $b\}$. If $(a, b) \in R$ define $a b=a \wedge b$. Notice by Lemma 3.3 if $(a, b),(b, c) \in R$ then $(a, b c) \in R$ if and only if $(a b, c) \in R$ and in such cases $a(b c)=a \wedge b \wedge c=(a b) c$. Thus $S_{1}(P)$ is a partial semigroup. Define $a^{*}=a$ for all $a \in P$ making $\left(S_{1}(P), R,{ }^{*}\right)$ a partial involution semigroup with zero 0 . Defining the projection corresponding to $a$ in condition (B) by $a^{\prime}$ we now show $\left(S_{1}(P), R,{ }^{*},{ }^{\prime}\right)$ is an OM-partial Baer *-semigroup. Notice that the following statements are equivalent. $(a, b) \in R$ and $a b=0 ; a \mathfrak{e} b$ and $a b=0 ; b=b \wedge a^{\prime} ;\left(a^{\prime}, b\right) \in R, a^{\prime} b=b$. Thus condition (B) holds. If $a \leqq b$ then $a \mathfrak{C} b$ so that $a^{\prime} \mathfrak{C} b$ and $\left(a^{\prime}, b\right) \in R$. Thus condition (OM) holds. It is clear that the identity map $a \rightarrow a$ gives the desired isomorphism from $P$ into $P^{\prime}\left(S_{1}(P)\right)$.

4. Embedding theorems. In the case of orthomodular lattices a coordinatizing Baer *-semigroup is not unique. Even "minimal" coordinatizing Baer *-semigroups may not be isomorphic. In our case, however, we do have a kind of uniqueness theorem. Let $S=\left(S, R,{ }^{*},{ }^{\prime}\right)$ and $S=\left(S, R, \#,{ }^{\circ}\right)$ be partial Baer ${ }^{*}$-semigroups. We say that $S$ can be embedded in $\delta$ if there is an injection $\tau: S \rightarrow S$ such that (i) $\tau R \subseteq R$, (ii) $\tau(x y)=(\tau x)(\tau y)$ for all $(x, y) \in R$, (iii) $\tau x^{*}=(\tau x) \#$ for all $x \in S$, (iv) $\tau x^{\prime}=(\tau x)^{\circ}$ for all $x \in S$.

Let $\varsigma_{0}=\varsigma_{0}(P)$ be the coordinatizing partial Baer *-semigroup constructed in Theorem 3.1. We now show that every coordinatizing partial Baer *-semigroup for $P$ “contains" $\delta_{0}$.

THEOREM 4.1. Let $P$ be an orthocomplemented poset and $S=\left(S, R, \#,{ }^{\circ}\right)$ 
a coordinatizing partial Baer *-semigroup for $P$. Then $\S_{0}(P)$ can be embedded in $\mathrm{s}$.

Proof. Let $\sigma: P \rightarrow P^{\prime}(\mathcal{S})$ be the coordinatizing isomorphism for $\mathcal{S}$ and define $\tau a=\sigma a$ for all $a \in S_{0}$. Then $\tau$ is an injection of $S_{0}$ onto $P^{\prime}(\mathcal{S})$. Now suppose $(a, b) \in R$ where of course $R$ is defined as in Theorem 3.1. If $a \mathrm{r} b$ then $\tau(a, b) \equiv(\tau a, \tau b)=(\sigma a, \sigma b) \in R$ and $\tau(a b)=\tau(a \wedge b)$ $=\sigma(a \wedge b)=(\sigma a)(\sigma b)=(\tau a)(\tau b)$. If $a \perp b$ then $\sigma b \leqq(\sigma a)^{\circ}$ and hence $(\sigma a)^{\circ}(\sigma b)=\sigma b$. Since $S$ is a partial Baer *-semigroup $(\sigma a, \sigma b) \in R$ and $(\sigma a)(\sigma b)=0$. So again $\tau(a, b) \in R$ and $\tau(a b)=\tau(0)=0=(\sigma a)(\sigma b)$ $=(\tau a)(\tau b)$. Finally $\tau\left(a^{*}\right)=\tau a=\sigma a=(\sigma a) \#=(\tau a) \#$ and $\tau\left(a^{\prime}\right)=\sigma\left(a^{\prime}\right)$ $=(\sigma a)^{\circ}=(\tau a)^{\circ}$.

Before we can get the analogue to Theorem 4.1 for orthomodular posets we need an additional result.

TheORem 4.2. Let $S$ be an OM-partial Baer *-semigroup and let e, $f \in P^{\prime}(S)$. Then $e$ e $f$ if and only if $(e, f) \in R$ and $e f=f e$.

Proof. Let $(e, f) \in R$ and $e f=f e$. Applying Lemmas 2.6 (ii) and 2.8 (ii) we have that $e \wedge f=e f=(e f)^{\prime \prime}=\left(e \bigvee f^{\prime}\right) \wedge f$. Also $(e, f) \in R$ implies by Lemma 2.8 (i) that $e^{\prime} \wedge f$ and $e \wedge f^{\prime}$ exist. Hence by Lemma 3.2 (1) we have $e \mathfrak{e} f$. Conversely, let $e \mathfrak{e} f$. We first show $(e, f) \in R$. $e$ e $f$ implies by definition that there exist mutually orthogonal elements $e_{1}, f_{1}, d \in P^{\prime}(S)$ such that $e=e_{1} \bigvee d$ and $f=f_{1} \bigvee d$. Therefore $e \leqq f_{1}^{\prime}$ and $\left(e, f_{1}^{\prime}\right) \in R$ with $e=e f_{1}^{\prime}$. Also $f_{1} \leqq f$ or $f_{1}=f_{1} f=f f_{1}$ which implies by $(\mathrm{OM})$ and Lemmas 2.6 (ii) and 2.7 that $f_{1}^{\prime} f=f f_{1}^{\prime}=f \wedge f_{1}^{\prime}=\left(f_{1} \vee d\right) \vee f_{1}^{\prime}=d \wedge f_{1}^{\prime}$ $=d$. Also $e=e_{1} \bigvee d \geqq d=f_{1}^{\prime} f$ says that $\left(e, f_{1}^{\prime} f\right) \in R$. Hence we have $\left(e, f_{1}^{\prime}\right)$, $\left(f_{1}^{\prime}, f\right),\left(e, f_{1}^{\prime} f\right) \in R$ from which $\left(e f_{1}^{\prime}, f\right)=(e, f) \in R$. By Lemma 2.8 (ii) and $3.2(1)$ we have $(e f)^{\prime \prime}=f \wedge e \leqq e$. Applying Lemma 2.2 ef $=(e f) e$ $=[(e f) e]^{*}=(e f)^{*}=f e$.

Corollary 4.3. Let $P$ be an orthomodular poset and let $S$ be a coordinatizing OM-partial Baer *-semigroup with coordinatizing isomorphism $\sigma$. If $a, b \in P$ then $a \mathfrak{C} b$ if and only if $(\sigma a, \sigma b) \in R$ and $(\sigma a)(\sigma b)$ $=(\sigma b)(\sigma a)$.

We also obtain the desired embedding theorem. We let $S_{1}=S_{1}(P)$ be the coordinatizing OM-partial Baer *-semigroup constructed in Theorem 3.4.

TheORem 4.4. Let $P$ be an orthomodular poset and $S=\left(S, R, \#,{ }^{\circ}\right)$ a coordinatizing OM-partial Baer *-semigroup for $P$. Then $S_{1}(P)$ can be embedded in $\mathrm{s}$.

Proof. Observe since $P \cong P^{\prime}(s)$ we have $S_{1}(P) \cong S_{1}\left(P^{\prime}(s)\right)$. Thus 
letting $R_{1}$ represent the related pairs in $S_{1}\left(P^{\prime}(\mathcal{S})\right) \subseteq \mathcal{S}$, Theorem 4.2 gives $R_{1} \subseteq \mathcal{Q}$ with the product in $S_{1}\left(P^{\prime}(\mathcal{S})\right.$ ) identical with that in $S$. Also \# and ${ }^{\circ}$ in $S_{1}\left(P^{\prime}(\mathcal{S})\right)$ are as in $\delta$ so the result is established.

Theorem 4.4 can also be proved using the method of Theorem 4.1 and conversely Theorem 4.1 can be proved using the method of Theorem 4.4.

\section{REFERENCES}

1. D. J. Foulis, Baer *-semigroups, Proc. Amer. Math. Soc. 11 (1960), 648-654. MR 23 \#A3105.

2. - Lecture notes on orthomodular lattices, University of Massachusetts, Amherst, Mass.

3. S. P. Gudder, "Axiomatic quantum mechanics and generalized probability theory," in Probabilistic methods in applied mathematics. Vol. II, A. Bharucha-Reid (Editor), Academic Press, New York, 1970.

4. V. S. Varadarajan, Probability in physics and a theorem on simultaneous observability, Comm. Pure Appl. Math. 15 (1962), 189-217; Erratum: Comm. Pure Appl. Math. 18 (1965), 757. MR 29 \#917; MR 32 \#8722.

University of Denver, Denver, Colorado 80210 and

Kansas State University, Manhattan, Kansas 66502 